\title{
Postauricular revolving door island flap: surgical option to concha squamous cell carcinoma
}

\begin{abstract}
The postauricular revolving door island flap is one versatile option that may be used to reconstruct the auricular concha of the ear after remove a squamous cell carcinoma. The technique has been described as an immediate one-stage elegant reconstructive procedure for closure of anterior auricular defects, simple and safe to perform with early excellent cosmetic results. A 71-year-old female Portuguese patient with Squamous Cell Carcinoma in left auricular concha and the external auditory canal is reported. Cutaneous squamous cell carcinoma is the second most common cancer of the skin in the United States. In the ear, this type of cancer because of its location is high risk and has a high possibility of metastasis. The tumor was completely excised including the cartilage. The surgical defect was reconstructed with Postauricular Revolving Door Island Flap based on the post auricular vessels. A full thickness design is made considering the defect, the flap is maintained to a subcutaneous central pedicle and is passed through the anterior window to resurface the anterior ear. This case demonstrates the use of the postauricular (revolving door) island pedicle flap for cover large anterior ear defects with loss of cartilaginous support and illustrates how the flap improve ear contour after resection successfully.
\end{abstract}

Keywords: auricular concha, trap door flap, flip-flop flap, ear squamous cell carcinoma, saloon door flap
Volume II Issue 6 - 2019

\author{
Casas J,, ${ }^{1,2}$ Pachano $\mathrm{O}^{1}$ \\ 'Department of Otolaryngology and Head \& Neck Surgery, \\ Luanda Medical Center, Angola \\ ${ }^{2}$ Department of Plastic Surgery, Luanda Medical Center,Angola
}

Correspondence: Casas J, Department of Plastic Surgery, Luanda Medical Center, Luanda,Angola,

Email jcasasve200@hotmail.com Received: December 04, 2019 | Published: December 30,
2019
Abbreviations: CSCC, Cutaneous squamous cell carcinoma; EAC, external auditory canal; SCCEAC, squamous cell carcinoma of the external auditory canal; NCCN, national comprehensive cancer network

\section{Introduction}

The ear because of its position is susceptible to trauma or exposure to ultraviolet light, this can result in ear damage, these injuries include lacerations, avulsions and cancer. Skin cancer is a frequent pathology, up to $25 \%$ of total cancers originate in skin: $90 \%$ of them are in the head and neck, and within this location $8 \%$ affects the external ear. Cutaneous squamous cell carcinoma (cSCC) is a malignant tumor of epidermal keratinocytes that invades the epidermis; local destruction can be extensive and in advanced stages it can metastasize. ${ }^{1}$ The external ear includes both the pinna and the external auditory canal (EAC). Squamous cell carcinoma of the pinna (SCCP) is considered a high-risk carcinoma that, because of its specific location, frequently presents recurrence and metastases. Squamous cell carcinoma of the external auditory canal (SCCEAC) is a very rare tumor. Invasion of cartilage by ( $\mathrm{CSCC})$ is rare and considered to be a risk factor for the development of metastatic disease., ${ }^{2,3}$ The National Comprehensive Cancer Network $(\mathrm{NCCN})$ guidelines recommend surgical excision with histologic confirmation of negative margins and close followup..$^{2,4}$

Clinically, it can occur in ulcerated, warty, exophytic or nodular form with irregular limits and in areas with greater exposure to the sun, which explains its greater frequency in the pinna than in the external auditory canal. ${ }^{5}$ The diagnosis is histopathological, since similar symptoms and signs make it difficult to infer the histological variety. The auricular skin cancers are located on the helix, antihelix and posterior ear, less frequently on the tragus, lobule or conchal cavity. ${ }^{6}$ Large anterior auricular defects are a reconstructive challenge due to complex topography of the ear and second intention healing can lead to poor cosmetic outcomes.

All this skin damage can be solved through various techniques such as grafts, local or regional flaps (postauricular pull-through flap, tubed pedicle flap, preauricular transposition flap and postauricular revolving door island flap), ${ }^{5,7}$ some have their disadvantages due to asymmetry of the ears, skin color of the donor area and sometimes require second surgeries (hematomas, chondritis, dehiscence, infections). The postauricular skin has similarities to the skin of the face, these qualities make it particularly attractive to be used as a donor area, either as grafts or as flaps of the upper or lower base to reconstruct the pinna and the external auditory canal.

One of these techniques developed and first described in 1972 by Masson, ${ }^{7}$ which has proven useful for reconstructing large conchal bowl defects, is the Revolving Door Island Flap. This procedure uses the skin of the postauricular and mastoid region as an island, rotates 180 degrees and goes under the conchal cavity defect, with primary closure of the defect in donor area. ${ }^{7-11}$ The surgery allows a good scarring without complications, ${ }^{6}$ both in the donor and recipient areas. This technique is used for defects of the conchal cavity and for lesions that require excision of the underlying cartilage, larger defects get better results. ${ }^{7,9,11}$ We describe a patient with a large conchal bowl defect, it was repaired by a postauricular revolving door (also known as a trap door or flip-flop ${ }^{10}$ island flap that provided skin coverage to the cavum conchae and restored the ear's structural integrity. The aim of this article was to describe our experience in the surgical procedure 
(postauricular island flap) which was used for reconstruction of central sites defects of the auricle, performed by the Otolaryngology and Plastic Surgery Department, we reviewed the technique and the advantages of reconstruction with this flap in a single surgical time.

\section{Case presentation}

A 71-year-old Portuguese female patient who claims to have a tumor in the left ear, which grows rapidly over a period of six months (2018) in the conchal surface without symptoms or signs of infections, who was treated at the Otolaryngology and Plastic Surgery Department of Luanda Medical Center (Luanda, Angola). The physical examination demonstrates an erythematous conchal cavity tumor, flaky surface and hyperkeratosis area that penetrates the left external auditory canal, without pain nor otorrhea (Figure 1). There is no lymphadenopathy on neck palpation, the patient is scheduled to undergo to a biopsy with local anesthesia.

Ears and mastoids tomography (Figure 2) are requested to evaluate the location and tumor extension, it is evident the left auricular tumor with extension to the ear canal. Neck CT scan was lymph nodes free. Patient returns two months later (Figure 3) to do the biopsy with local anesthesia, a greater erythematous tumor size is evident, already occupies the entire conchal bowl and obstructs the left auditory meatus, with a hyperkeratosis center, round edges and small telangiectasias area. The biopsy reports Keratoacanthoma, the similarity between Squamous Cell Carcinoma and Keratoacanthoma is well known and makes a difficult pathological diagnosis, therefore we underwent with tumor excision with oncologic margins (Figures 4). Oncologic resection was complete, includes skin and cartilage (Figures 4). Once the tumor is carefully excised it is sent for pathological analysis and diagnosis. The flap reconstruction (posterior island flap) begins once the tumor excision culminates, taking into consideration the size of the tumor and its difficult approach.

The postauricular pedicle island flap was used for repaired defects

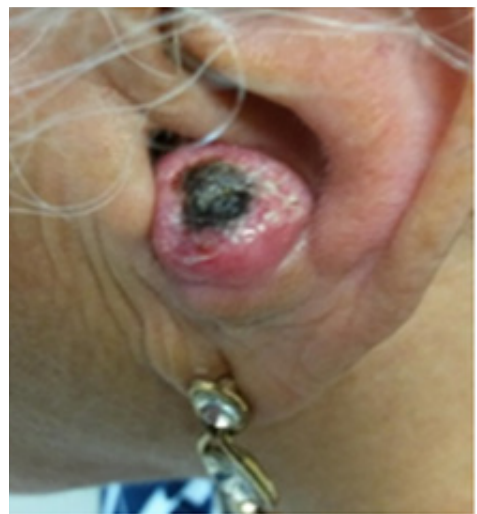

Figure I Round edges lesion of $2 \mathrm{~cm}$ of diameter, erythematous with hyperkeratotic center pigmented on the surface that completely obliterates the meatus of the external auditory canal.

located in the concha. The vertical axis of the flap was created along the sulcus auriculae posterior. The tissue of the pedicle island flap was created to be larger than the area of the defect. The length of the pedicle was approximately $2 \mathrm{~cm}$. A skin incision of the posterior auricle was made just below the helical rim, taking care to preserve the skin over the pedicle. The flap was tongue shaped with a length to width ratio of 4:1. A wide slit was created along the postauricular sulcus to allow the flap pass without resistance and reducing the risk of ischemia, the flap was pulled through the slit onto the anterior surface of the concha. The postauricular donor site was closed through primary skin closure. The patient was discharged after showing good recovery and the sutures were removed one week later. Complete healing occurred in 15 days without complications (dehiscence, necrosis, hematoma, infection), 18 - months follow-up revealed no recurrences and complete patient satisfaction with the aesthetic outcome (Figure 5). This very versatile flap allows a wide coverage and gives good postoperative aesthetic results. The histopathological report: Well-differentiated Squamous Cell Carcinoma, with negative margins.
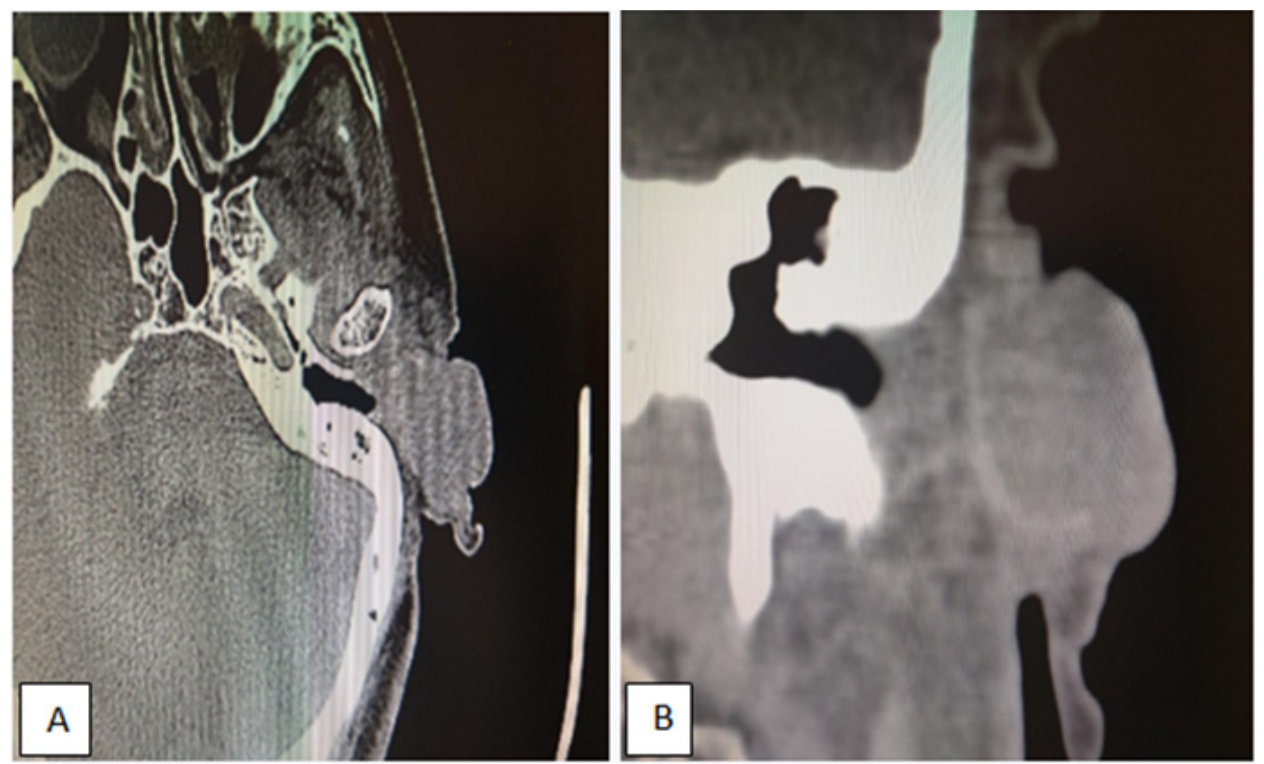

Figure 2 Axial computed tomography, (A) it demonstrated left ear hypodense expansive tissue with inadequate contrast capture. (B) Shows conchal bowl soft tissue that occupies the anterior third of the external auditory canal without bone infiltration. 

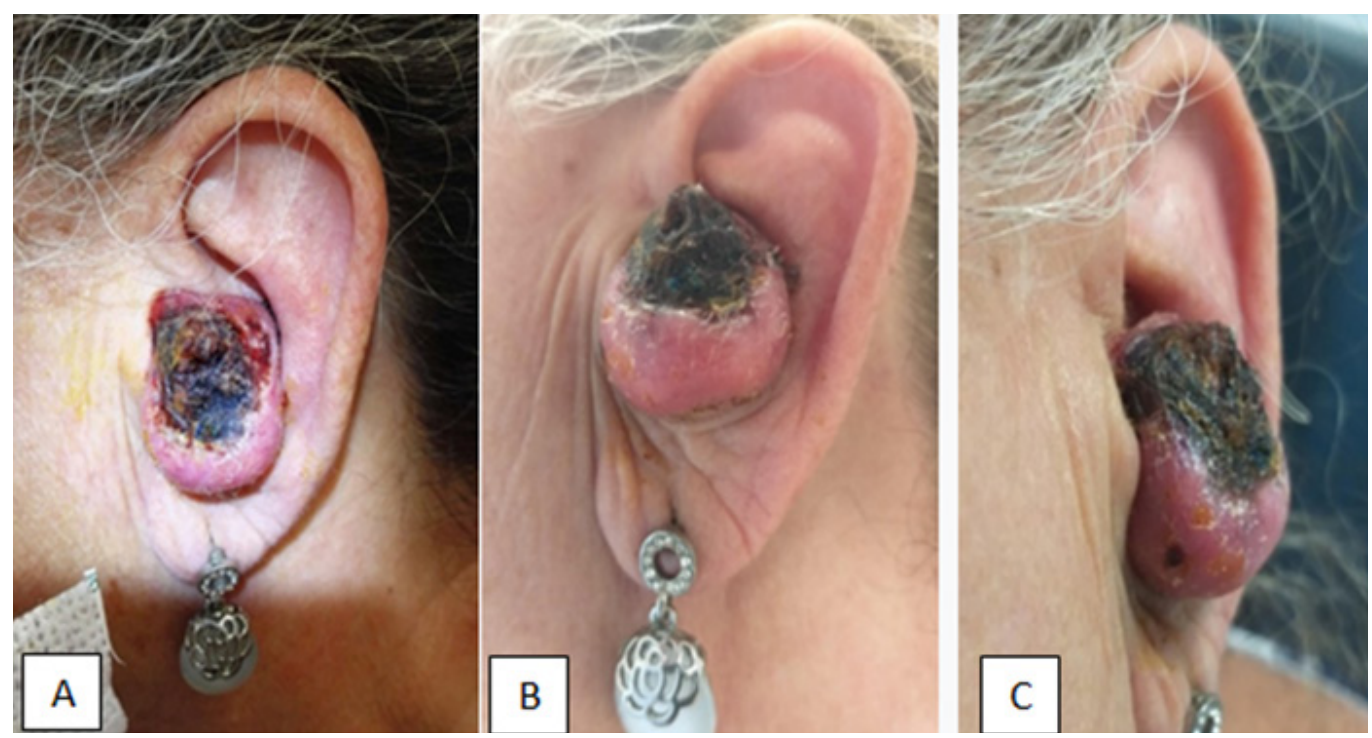

Figure 3 Two months later the tumor grows rapidly (before surgery), round erythematous and hyperkeratotic tumor. (A) Frontal view; (B) Inferior view; (C) Lateral view.
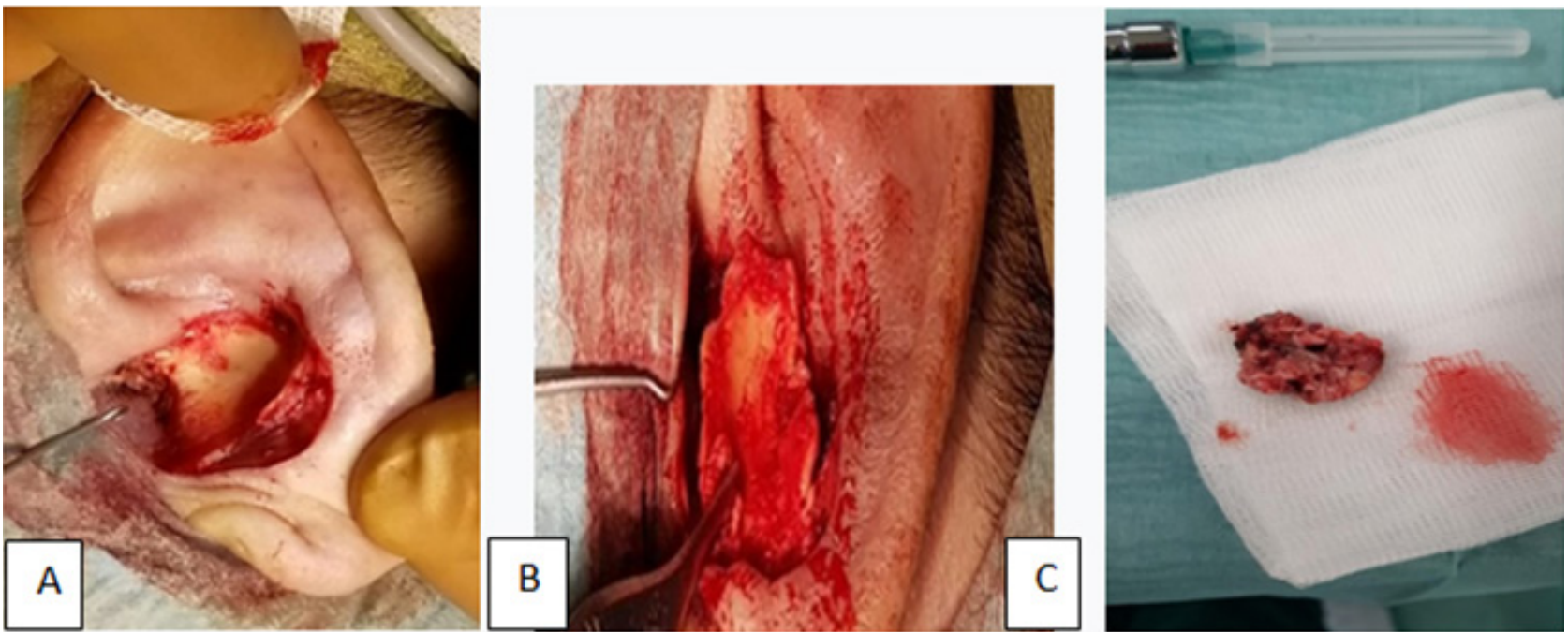

Figure 4 A) Left ear after tumor resection, cartilage in the background; (B) Left auricular concha cartilage dissected; (C) Left ear concha tumor removed.

\section{Discussion}

The pinna and the external auditory canal are seat of various pathologies. Among the malignant tumor pathologies, the most frequent is Epidermoid Cell Carcinoma in external ear, which when detected in early stages allows its resection without major complications. Reconstruction of the auricular concha cavity is difficult because it's a unique structure. There are a variety of surgical procedures to be used to reconstruct the result of these pathologies such as grafts and flaps (preauricular transposition flap, tubed pedicle flap, postauricular pull-through flap and postauricular revolving door island flap). ${ }^{4}$ Postauricular flaps have proved effective in the reconstruction of the auricular defects. However, few reports exist regarding the reconstruction of centrally defects of the concha cavity. ${ }^{12}$ The postauricular revolving door island flap was the best option for our patient because of its one-stage reconstruction, color, texture and thickness match, its proximity to the defect and result was cosmetic and functionally excellent for the patient. ${ }^{13}$

\section{Conclusion}

The postauricular skin has similarities to the skin of the face, these qualities make it particularly attractive to be used as a donor area. The postauricular pedicle flap is well suited for one stage reconstruction of anterior auricular defects lacking perichondrium without additional donor site deformity, low morbidity and satisfactory outcomes. 

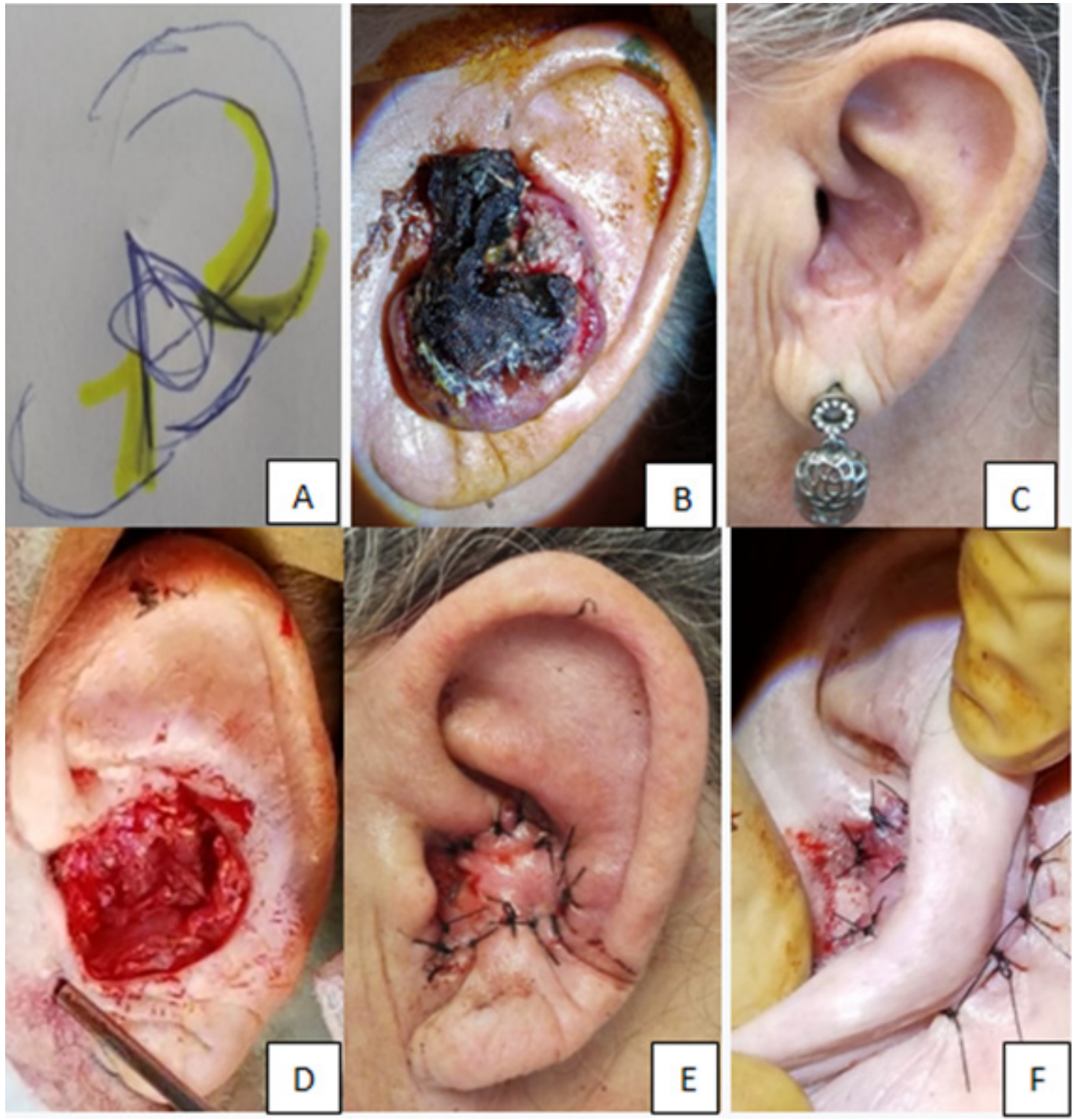

Figure 5 (A) Postauricular revolving door island flap design; (B) Before surgery; (C) 3 months after surgery; (D) Ear after tumor resection; (E) Frontal projection at the end of surgery; (F) Anterior and posterior interrupted suture pattern.

\section{Consent}

The consent was obtained from the patient for the publication of the case presentation.

\section{Ethical approval}

This study was reviewed and approved by the Institutional Review Board of the Luanda Medical Center. The investigation of this case was conducted on the principles of the Declaration of Helsinki.

\section{Acknowledgements}

Julio Casas Ocando and Oscar Pachano Marquez contributed equally to the work.

\section{Conflicts of interest}

There are no conflicts of interest.

\section{References}

1. Wells GL, West A. Carcinoma espinocelular-Trastornos cutáneosManual MSD.

2. Clark RR, Soutar DS, Hunter KD.A retrospective analysis of histological prognostic factors for the development of lymph node metastases from auricular squamous cell carcinoma. Histology. 20 I0;57(I): 138-I46.

3. Boisen J, Malone H, Brent K, et al. Cutaneous Squamous cell carcinoma with invasion through ear cartilage. Case Rep Dermatol Med. 2016;2016:9067428. 
4. National Comprehensive Cancer Network (NCCN) NCCN Clinica Practice Guidelines in Oncology (NCCN Guidelines). Squamous Cell Carcinoma. 20I4.

5. García E, Di Lella F, Larrañaga J, et al. Carcinoma espinocelular del oído externo con extension parotídea: a propósito de un caso. Rev Hosp ltal B Aires. 20 16;36(2):53-58.

6. Vuyk $\mathrm{H}$, Cook T.Auricular reconstruction after Mohs surgery: a review. FACE. 1997;5(I):9-2I.

7. Humphhreys TR, Goldberg LH,Wiemer DR. The postauricular (revolving door) island pedicle flap revisited. Dermatologic Surgery. I996;22(2): I48150.

8. Masson JK.A simple flap for reconstruction of concha helix defects. $B R$ J Plast Surg. 1972;25:399.

9. Russo de la Torre F. Retroauricular revolving door island flap. Actas Dermosifiliorg. 20I2; 103(8):737-738.
10. Nemir S, Hunter-Ellul L, Codrea V, et al. Reconstruction of a large anterior ear defect after Mohs micrographic surgery with a cartilage graft and postauricular revolving door flap. Case Rep Dermatol Med. 2015;2015:4848I9.

11. Redondo P, Lloret P, Sierra A, et al. Aggressive tumor of the concha: treatment with postauricular island pedicle flap. J Cutan Med Surg. 2003;7(4):339-43.

12. Papadiochos I, Bourlidou E, Mangoudi D. A simplified reconstructive technique for full-thickness central defects of the auricle with the use of post-auricular fold flap.J Cutan Aesthet Surg. 2017; 10(2): I09-II 2.

13. Dessy LA, FigusA, Fioramonti P, et al. Reconstruction of anterior auricular conchal defect after malignancy excision: revolving-door flap versus fullthickness skin graft.J Plast Reconstr Aesthet Surg. 2010;63(5):746-752. 